\title{
Vestibular migraine: Diagnostic criteria
}

\author{
Consensus document of the Bárány Society and the International Headache Society
}

\author{
Thomas Lempert $^{\mathrm{a}, *}$, Jes Olesen ${ }^{\mathrm{b}}$, Joseph Furman ${ }^{\mathrm{c}}$, John Waterston $^{\mathrm{d}}$, Barry Seemungal ${ }^{\mathrm{e}}$, John Carey $^{\mathrm{f}}$, \\ Alexander Bisdorff ${ }^{g}$, Maurizio Versino ${ }^{\mathrm{h}}$, Stefan Evers ${ }^{\mathrm{i}}$ and David Newman-Toker ${ }^{\mathrm{j}}$ \\ ${ }^{a}$ Department of Neurology, Schlosspark-Klinik, Berlin, Germany \\ ${ }^{\mathrm{b}}$ Danish Headache Center and Department of Neurology, University of Copenhagen, Copenhagen, Denmark \\ ${ }^{\mathrm{c}}$ Departments of Otolaryngology and Neurology, University of Pittsburgh, Pittsburgh, PA, USA \\ ${ }^{\mathrm{d}}$ Department of Neurology and Monash University Department of Medicine, Alfred Hospital, Melbourne, Australia \\ ${ }^{\mathrm{e}}$ Department of Clinical Neuroscience, Charing Cross Hospital, London, UK \\ ${ }_{\mathrm{f}}^{\mathrm{f}}$ Department of Otolaryngology, Head and Neck Surgery, Johns Hopkins University School of Medicine, Baltimore, \\ $M D, U S A$ \\ ${ }^{g}$ Department of Neurology, Centre Hospitalier Emile Mayrisch, Esch-sur-Alzette, Luxembourg \\ ${ }^{\mathrm{h}}$ Department of Neurological Sciences University of Pavia, HSC and BCC National Neurological Institute IRCCS \\ C. Mondino Foundation, Pavia, Lombardy, Italy \\ i Department of Neurology, University of Münster, Münster, Germany \\ ${ }^{\mathrm{j}}$ Department of Neurology, Johns Hopkins University School of Medicine, Baltimore, MD, USA
}

\begin{abstract}
This paper presents diagnostic criteria for vestibular migraine, jointly formulated by the Committee for Classification of Vestibular Disorders of the Bárány Society and the Migraine Classification Subcommittee of the International Headache Society (IHS). The classification includes vestibular migraine and probable vestibular migraine. Vestibular migraine will appear in an appendix of the third edition of the International Classification of Headache Disorders (ICHD) as a first step for new entities, in accordance with the usual IHS procedures. Probable vestibular migraine may be included in a later version of the ICHD, when further evidence has been accumulated. The diagnosis of vestibular migraine is based on recurrent vestibular symptoms, a history of migraine, a temporal association between vestibular symptoms and migraine symptoms and exclusion of other causes of vestibular symptoms. Symptoms that qualify for a diagnosis of vestibular migraine include various types of vertigo as well as head motion-induced dizziness with nausea. Symptoms must be of moderate or severe intensity. Duration of acute episodes is limited to a window of between 5 minutes and 72 hours.
\end{abstract}

Keywords: Migraine, vertigo, dizziness, vestibular, diagnostic criteria, Bárány Society, International Headache Society

\section{Introduction}

The link between migraine and vertigo was recognized by some of the early neurologists in the 19th century [1], but systematic studies of vertigo caused by migraine started only a hundred years later [2,3].

\footnotetext{
* Corresponding author: Thomas Lempert, Department of Neurology, Schlosspark-Klinik, Berlin, Germany. E-mail: Thomas. Lempert@schlosspark-klinik.de.
}

In the past three decades vestibular migraine has taken shape as a diagnostic entity that may affect up to $1 \%$ of the general population [4]. Despite this recognition, the lack of a universally accepted definition of vestibular migraine has hampered patient identification in clinical and research settings. In response, the Bárány Society, which represents the international community of basic scientists, otolaryngologists and neurologists committed to vestibular research, mandated a classification group to develop diagnostic criteria for vestibular migraine. The definition of vestibular migraine is part of 
a larger endeavor for classification of neuro-otological disorders steered by the Committee for Classification of Vestibular Disorders of the Bárány Society. Individual disorders are defined by classification groups which include otolaryngologists and neurologists from at least three continents. The format of the classification is modeled on the International Classification of Headache Disorders. As a first step and prerequisite for the classification of vestibular disorders, the Classification Committee of the Bárány Society published a consensus on the definitions of vestibular symptoms [5].

A principal aim of the definition of vestibular migraine is its broad acceptance within both the vestibular and headache communities. Therefore, drafts of this classification were discussed with the Migraine Classification Subcommittee of the Classification Committee of the International Headache Society (IHS). Controversies centered on the balancing of the sensitivity and specificity of the criteria. This conflict is inherent to all medical classification efforts, because highly specific criteria will inevitably exclude patients affected by the condition (false negatives), while highly sensitive criteria will include patients who do not have the condition (false positives). As a result of these discussions, a proposed category, possible vestibular migraine, was eliminated while the categories vestibular migraine and probable vestibular migraine were retained. Other changes that resulted from discussions between the Bárány Society and the IHS included a narrower definition of the duration of acute episodes and a sharpened focus on the careful exclusion of differential diagnoses.

The final approved diagnostic criteria were the product of an accord between the IHS Classification Committee and the Committee for Classification of Vestibular Disorders of the Bárány Society. These criteria will be included in the 3rd edition of the International Classification of Headache Disorders, expected to be published in 2014, where it will appear in the appendix for new disorders that need further research for validation. In addition, the classification of vestibular migraine is part of the evolving Classification of Vestibular Disorders of the Bárány Society. The new ICHD-3 will only include vestibular migraine, while the Bárány classification also will contain probable vestibular migraine.

\section{Diagnostic criteria for vestibular migraine}

Previously used terms: migraine-associated vertigo/ dizziness, migraine-related vestibulopathy, migrainous vertigo.
(For the relationship between vestibular migraine and basilar-type migraine (migraine with brainstem au$r a$ according to ICHD-3) and the relationship between vestibular migraine and benign paroxysmal vertigo of childhood, please see comments.)

\section{Vestibular migraine}

A. At least 5 episodes with vestibular symptoms ${ }^{1}$ of moderate or severe intensity ${ }^{2}$, lasting $5 \mathrm{~min}$ to 72 hours $^{3}$

B. Current or previous history of migraine with or without aura according to the International Classification of Headache Disorders (ICHD) ${ }^{4}$

C. One or more migraine features with at least $50 \%$ of the vestibular episodes ${ }^{5}$ :

- headache with at least two of the following characteristics: one sided location, pulsating quality, moderate or severe pain intensity, aggravation by routine physical activity

- photophobia and phonophobia ${ }^{6}$,

- visual aura ${ }^{7}$

D. Not better accounted for by another vestibular or ICHD diagnosis ${ }^{8}$

\section{Probable vestibular migraine}

A. At least 5 episodes with vestibular symptoms ${ }^{1}$ of moderate or severe intensity ${ }^{2}$, lasting $5 \mathrm{~min}$ to 72 hours $^{3}$

B. Only one of the criteria $\mathrm{B}$ and $\mathrm{C}$ for vestibular migraine is fulfilled (migraine history or migraine features during the episode)

C. Not better accounted for by another vestibular or ICHD diagnosis ${ }^{8}$

\subsection{Notes}

1. Vestibular symptoms, as defined by the Bárány Society's Classification of Vestibular Symptoms [5] and qualifying for a diagnosis of vestibular migraine, include:

- spontaneous vertigo including

* internal vertigo, a false sensation of selfmotion, and

* external vertigo, a false sensation that the visual surround is spinning or flowing,

- positional vertigo, occurring after a change of head position,

- visually-induced vertigo, triggered by a complex or large moving visual stimulus

- head motion-induced vertigo, occurring during head motion, 
- head motion-induced dizziness with nausea. Dizziness is characterized by a sensation of disturbed spatial orientation. Other forms of dizziness are currently not included in the classification of vestibular migraine.

2. Vestibular symptoms are rated "moderate" when they interfere with but do not prohibit daily activities and "severe" if daily activities cannot be continued.

3. Duration of episodes is highly variable: About $30 \%$ of patients have episodes lasting minutes, $30 \%$ have attacks for hours and another $30 \%$ have attacks over several days. The remaining $10 \%$ have attacks lasting seconds only, which tend to occur repeatedly during head motion, visual stimulation, or after changes of head position. In these patients, episode duration is defined as the total period during which short attacks recur. At the other end of the spectrum, there are patients who may take four weeks to fully recover from an episode. However, the core episode rarely exceeds 72 hours [6-11].

4. Migraine categories 1.1 and 1.2 of the ICDH [12].

5. One symptom is sufficient during a single episode. Different symptoms may occur during different episodes. Associated symptoms may occur before, during or after the vestibular symptoms.

6. Phonophobia is defined as sound-induced discomfort. It is a transient and bilateral phenomenon that must be differentiated from recruitment, which is often unilateral and persistent. Recruitment leads to an enhanced perception and often distortion of loud sounds in an ear with decreased hearing.

7. Visual auras are characterized by bright scintillating lights or zigzag lines, often with a scotoma that interferes with reading. Visual auras typically expand over 5-20 minutes and last for less than 60 minutes. They are often, but not always restricted to one hemifield. Other types of migraine aura, e.g. somatosensory or dysphasic aura, are not included as diagnostic criteria because their phenomenology is less specific and most patients also have visual auras.

8. History and physical examinations do not suggest another vestibular disorder or such a disorder is considered but ruled out by appropriate investigations or such disorder is present as a comorbid or independent condition, but episodes can be clearly differentiated. Migraine attacks may be induced by vestibular stimulation [13]. Therefore, the differential diagnosis should include other vestibular disorders complicated by superimposed migraine attacks. See Comments for discussion of specific differential diagnoses.

\section{Comments}

\subsection{Epidemiology}

Vestibular migraine is one of the most common vestibular disorders affecting up to $1 \%$ of the general population [4] and $11 \%$ of patients in specialized dizziness clinics [6]. Familial occurrence of vestibular migraine with autosomal dominant inheritance has been documented in several families [14].

\subsection{Relation to migraine aura and basilar-type migraine}

Both migraine aura and basilar-type migraine are terms defined by the ICDH-2 [12]. Only a minority of patients with vestibular migraine experience their vertigo in the time frame of 5-60 minutes as defined for an aura. Even fewer have their vertigo immediately before headache starts, as required for the ICDH-2 category typical aura with migraine headache [12]. Therefore, episodes of vestibular migraine cannot be regarded as migraine auras.

Although more than $60 \%$ of basilar-type migraine patients have vertigo, the ICDH-2 requires at least two posterior circulation manifestations lasting between 5 and 60 minutes, followed by a migraine headache for a diagnosis of basilar-type migraine. Less than $10 \%$ of patients with vestibular migraine fulfill these criteria [6, 7]. Thus, vestibular migraine and basilar-type migraine are not synonymous, although individual patients may meet the diagnostic criteria for both conditions. Further studies are needed to define overlap and delineation of the two conditions.

\subsection{Relation to benign paroxysmal vertigo of childhood}

While vestibular migraine may start at all ages, the ICHD specifically recognizes an early manifestation called benign paroxysmal vertigo of childhood. The diagnosis requires five episodes of severe vertigo, occurring without warning and resolving spontaneously after minutes to hours. In between episodes, neurological ex- 
amination, audiometry, vestibular functions and EEG must be normal [12]. A unilateral throbbing headache may occur during attacks but is not a mandatory criterion. Benign paroxysmal vertigo of childhood is regarded as one of the precursor syndromes of migraine. Therefore, previous migraine headaches are not required for diagnosis. Since the classification of vestibular migraine does not involve any age limit, the diagnosis can be applied in children when the respective criteria are met. Only children with different types of vertigo attacks, e.g. short ones lasting less than 5 minutes and longer ones lasting more than five minutes, should receive both diagnoses.

\subsection{Other symptoms}

Transient auditory symptoms [7], nausea, vomiting, prostration, and susceptibility to motion sickness may be associated with vestibular migraine. However, as they also occur with various other vestibular disorders they are not included as diagnostic criteria.

\subsection{Results of vestibular testing}

Vestibular migraine is classified entirely on the basis of clinical features as reported by the patient. Just as in migraine itself, there are no biological markers for vestibular migraine. Vestibular findings and testing results can be pathological, particularly during or shortly after an episode $[15,16]$, but they are not sufficiently specific to serve as diagnostic criteria. Profound abnormalities in the symptom-free interval such as severe hearing loss and complete unilateral or bilateral vestibular loss are usually indicative of another cause.

\subsection{Overlap with Menière's disease}

Migraine is more common in patients with Menière's disease than in healthy controls [17]. Patients with features of both Menière's disease and vestibular migraine have been repeatedly reported [17,18]. In fact, migraine and Menière's disease can be inherited as a symptom cluster [19]. Fluctuating hearing loss, tinnitus and aural pressure may occur in vestibular migraine, but hearing loss does not progress to profound levels $[7,20]$. Similarly, migraine headaches, photophobia and even migraine auras are common during Menière attacks [17,21]. The pathophysiological relationship between vestibular migraine and Menière's disease remains uncertain. In the first year after onset of symptoms, differentiation of vestibular migraine from
Menière's disease may be challenging, as Menière's disease can be monosymptomatic with vestibular symptoms only in the early stages of the disease.

When the criteria for Menière's disease [22] are met, particularly hearing loss as documented by audiometry, Menière's disease should be diagnosed, even if migraine symptoms occur during the vestibular attacks. Only patients who have two different types of attacks, one fulfilling the criteria for vestibular migraine and the other for Menière's disease, should be diagnosed with the two disorders. A future revision of this classification may include a vestibular migraine/Menière's disease overlap syndrome [23].

\subsection{Other differential diagnoses}

\subsubsection{Benign paroxysmal positional vertigo (BPPV)}

Vestibular migraine may present with purely positional vertigo, thus mimicking BPPV. Direct nystagmus observation during the acute phase may be required for differentiation. In vestibular migraine, positional nystagmus is usually persistent and not aligned with a single semicircular canal. Symptomatic episodes tend to be shorter with vestibular migraine (minutes to days rather than weeks) and more frequent (several times per year with vestibular migraine rather than once every few years with BPPV) [24].

\subsubsection{Transient ischemic attacks (TIAs)}

A differential diagnosis of vertebrobasilar TIAs must be considered particularly in elderly patients. Suggestive features include vascular risk factors, sudden onset of symptoms, total history of attacks of less than one year, and angiographic or Doppler ultrasound evidence for vascular pathology in the vertebral or proximal basilar artery.

\subsubsection{Vestibular paroxysmia}

Vestibular paroxysmia is a controversial disorder, presumably caused by vascular compression of the vestibular nerve. The presenting feature is brief attacks of vertigo, lasting from one to several seconds, which recur many times per day. Successful prevention of attacks with carbamazepine supports the diagnosis.

\subsubsection{Psychiatric dizziness}

Anxiety and depression may cause dizziness and likewise complicate a vestibular disorder. Anxietyrelated dizziness is characterized by situational provocation, intense autonomic activation, catastrophic thinking and avoidance behaviour. More than $50 \%$ of patients with vestibular migraine have comorbid psychiatric disorders [25]. 


\subsection{Migraine induced by vestibular activation}

Caloric stimulation often triggers migraine attacks within 24 hours in patients with migraine [13], which shows that migraine attacks can be a secondary effect of vestibular activation rather than its cause in susceptible individuals. Possibly, the high rate of headaches and other migraine symptoms during Menière attacks can be explained by this mechanism. Thus, migraine symptoms during vertigo attacks do not prove a diagnosis of vestibular migraine and consideration of other potential causes remains mandatory.

\subsection{Provoking factors}

Provocation of an episode can be a diagnostic clue. Menstruation, stress, lack of sleep, dehydration, and certain foods may all trigger migraine attacks, but are not included as diagnostic criteria for vestibular migraine because their sensitivity and specificity have not been adequately studied.

\subsection{Response to antimigraine medication}

A favorable response to anti-migraine drugs may support the suspicion of an underlying migraine mechanism. However, the apparent efficacy of a drug may be influenced by confounding factors including spontaneous improvement, placebo response, and multiple drug effects (e.g. anxiolytic or antidepressant). Also, drug responses are useful for diagnosis only when the sensitivity and specificity of the criterion is high. So far, the evidence for treating vestibular migraine with anti-migraine drugs is insufficient as it is based on uncontrolled clinical case series rather than randomized controlled trials [26]. Consequently, a positive drug response is not regarded as a reliable criterion for the diagnosis of vestibular migraine.

\subsection{Chronic vestibular migraine}

In this classification, vestibular migraine is conceptualized as an episodic disorder. However, a chronic variant of vestibular migraine has been reported [10, 27]. A distinction between chronic vestibular migraine and comorbid psychiatric dizziness syndromes seems particularly challenging in these patients [28]. In the future, following additional research, chronic vestibular migraine may become a formally recognized category of a revised classification.

\section{Acknowledgements}

This work was supported by travelling grants from the Bárány Society and from Neuro+ Berlin, a nonprofit association for neurological research. Thomas Lempert wishes to thank Hanne Neuhauser, Andrea Radtke and Michael von Brevern for many fruitful discussions on the classification of vestibular migraine.

\section{References}

[1] E. Liveing, On megrim: sick headache and some allied health disorders: a contribution to the pathology of nerve storms, London (1873), 129-148.

[2] A. Kuritzky, D.K. Ziegler and R. Hassanein, Vertigo, motion sickness and migraine, Headache 21 (1981), 227-231.

[3] A. Kayan and J.D. Hood, Neuro-otological manifestations of migraine, Brain 107 (1984), 1123-1142.

[4] H. Neuhauser, A. Radtke, M. von Brevern, M. Feldmann, F. Lezius, T. Ziese and T. Lempert, Migrainous vertigo: prevalence and impact on quality of life, Neurology 67 (2006), 1028-1033.

[5] A. Bisdorff, M. von Brevern, T. Lempert and D.E. NewmanToker (on behalf of the Committee for the Classification of Vestibular Disorders of the Bárány Society), Classification of vestibular symptoms: Towards an international classification of vestibular disorders, J Vest Res 19 (2009), 1-13.

[6] H. Neuhauser, M. Leopold, M. von Brevern, G. Arnold and T. Lempert, The interrelations of migraine, vertigo, and migrainous vertigo, Neurology 56 (2001), 436-441.

[7] G.D. Johnson, Medical management of migraine-related dizziness and vertigo, Laryngoscope 108 (1998), 1-28.

[8] M. Dieterich and T. Brandt, Episodic vertigo related to migraine (90 cases): vestibular migraine? J Neurol 246 (1999), 883-892.

[9] F.M.Cutrer and R.W. Baloh, Migraine-associated dizziness, Headache 32 (1992), 300-304.

[10] S.P.Cass, J.K.P. Ankerstjerne, S. Yetiser, J. Furman, C. Balaban and B. Aydogan, Migraine- related vestibulopathy, Ann Otol Rhinol Laryngol 106 (1997), 182-189.

[11] M. Versino and G. Sances, Dizziness and migraine: a causal relationship? Funct Neurol 18 (2003), 97-101.

[12] International Headache Society Classification Subcommittee, International Classification of Headache Disorders. 2nd Edition, Cephalalgia 24(Suppl 1) (2004), 1-160. (www.ihsclassification.org/en/).

[13] L. Murdin, R.A. Davies and A. Bronstein, Vertigo as a migraine trigger, Neurology 73 (2009), 638-642.

[14] A.K. Oh, H. Lee, J.C. Jen, S. Corona, K.M. Jacobson and R.W. Baloh, Familial benign recurrent vertigo, Am J Med Genet 100 (2001), 287-291.

[15] M. von Brevern, D. Zeise, H. Neuhauser, A.H. Clarke and T. Lempert, Acute migrainous vertigo: clinical and oculographic findings, Brain 128 (2005), 365-374.

[16] S. Hartman Polensek and R. Tusa, Nystagmus during attacks of vestibular migraine: An aid in diagnosis, Audiol Neurotol 15 (2010), 241-246.

[17] A. Radtke, T. Lempert, M.A. Gresty, G.B.Brookes, A.M. Bronstein and H. Neuhauser, Migraine and Menière's disease: is there a link? Neurology 59 (2002), 1700-1704. 
[18] Y.H. Cha, J. Brodsky, G.Ishiyama, C. Sabatti and R.W. Baloh, The relevance of migraine in patients with Meńière's disease, Acta Otolaryngol 127 (2007), 1241-1245.

[19] Y.H. Cha, M.J. Kane and R.W. Baloh, Familial clustering of migraine, episodic vertigo, and Ménière's disease, Otol Neurotol 29 (2008), 93-96.

[20] A. Radtke, H. Neuhauser, M. von Brevern, T. Hottenrott and T. Lempert, Vestibular migraine - validity of clinical diagnostic criteria, Cephalalgia 31 (2011), 906-913.

[21] K. Brantberg and R.W. Baloh, Similarity of vertigo attacks due to Meniere's disease and benign recurrent vertigo both with and without migraine, Acta Otolaryngol 131 (2011), 722-727.

[22] Committee on Hearing and Equilibrium guidelines for the diagnosis and evaluation of therapy in Menière's disease. American Academy of Otolaryngology - Head and Neck Foundation, Otolaryngol Head Neck Surg 113 (1995), 181-185.

[23] B.A. Neff, J.P. Staab, S.D. Eggers, M.L. Carlson, W.R. Schmitt, K.M. Van Abel, D.K. Worthington, CW. Beatty, C.L. Driscoll and N.T. Shepard, Auditory and vestibular symptoms and chronic subjective dizziness in patients with Menière's disease, vestibular migraine, and Menière's disease with concomitant vestibular migraine, Otol Neurotol 33 (2012) 12351241.

[24] M. von Brevern, A. Radtke, A. Clarke and T. Lempert, Migrainous vertigo presenting as episodic positional vertigo, Neurology 62 (2004), 469-472.

25] C. Best, R. Tschan, A. Eckhardt-Henn and M. Dieterich, Who is at risk for ongoing dizziness and psychological strain after a vestibular disorder? Neuroscience 164 (2009), 1579-1587.

[26] M. Fotuhi, B. Glaun, S.Y. Quan and T. Sofare, Vestibular migraine: a critical review of treatment trials, J Neurol 256 (2009), 711-716.

[27] J. Waterston, Chronic migrainous vertigo, J Clin Neurosci 11 (2004), 384-388.

[28] J.M. Furman, C.D. Balaban, R.G. Jacob and D.A. Marcus, Migraine-anxiety related dizziness (MARD) : A new disorder? J Neurol Neurosurg Psychiat 76 (2005), 1-8. 